\title{
El impacto de la crisis política y económica sobre la polarización de los medios españoles
}

\author{
Laura TERUEL RODRÍGUEZ \\ Universidad de Málaga \\ teruel@uma.es
}

Recibido: 11 de febrero de 2015

Aceptado: 25 de febrero de 2016

\begin{abstract}
Resumen
En este trabajo se analiza la polarización que caracteriza a la prensa española desde los años 90 para, a partir de ello, realizar un análisis prospectivo que plantea algunas dudas sobre su pervivencia en los mismos términos dado el actual contexto crisis política y económica. Se persigue definir el rol desempeñado por los actores políticos y mediáticos centrándonos en las legislaturas en las que la crispación alcanzó mayores cotas (1993-1996 y 2004-2008) y trazar las novedades que afronta este escenario desde 2011, periodo en el que el debate público se encuentra fuertemente polarizado a pesar de que existen notables diferencias -políticas, sociales o económicas- con las anteriores etapas. Para ello, se ha realizado un extenso análisis de los contenidos mediáticos, de fuentes primarias de investigación sociológica y de la literatura científica sobre la polarización política. Se trata de un análisis de casos exploratorio, descriptivo y crítico.
\end{abstract}

Palabras clave: política; polarización; prensa; España; periódicos; televisión.

\section{The impact of political and economic crisis on the polarization of the Spanish media}

\begin{abstract}
In this paper the characteristics of the cyclical political polarization of the Spanish media system are defined. From this study, a prospective analysis raises doubts about this scenario remains unchanged because of the political and economic crisis. It seeks to define the role played by political and media actors in polarization focusing on the two legislatures where the tension reached higher levels (19931996 and 2004-2008) and compares it with the developments faced by them in the current economical and political context of crisis. To achieve these aims, it has been performed an analysis of media content (since 1993) and looked through primary sociological sources and the scientific literature about polarization. This is an exploratory, critical and descriptive case analysis.
\end{abstract}

Keywords: politics; polarization; press; Spain; newspapers; television.

\section{Referencia normalizada}

Teruel Rodríguez, L. (2016). El impacto de la crisis política y económica sobre la polarización de los medios españoles. Historia y Comunicación Social. Vol 21, número 1, páginas 203-220.

Sumario: 1. Introducción. 2 Marco teórico. Conceptualizando la polarización en el escenario político y mediático español. 3. Metodología. 4. Análisis: Características endémicas de la crispación mediática española desde los años noventa hasta 2008. 5. La polarización en el escenario de crisis política y mediática a partir de 2011. 6. Conclusiones. 7. Referencias bibliográficas. 


\section{Introducción}

En los contextos de crisis, como el actual, pueden observarse con nitidez las características del modelo de medios pluralista polarizado propio de España (Hallin y Mancini, 2003). Un sistema que se define por una prensa de elite con una circulación reducida, mayor consumo audiovisual y medios que presentan un alto grado de paralelismo político. De esta forma, el pluralismo se manifiesta en su vertiente 'externa' -cada medio se alinea con una propuesta ideológica y de forma sólida, presenta gran homogeneidad en su discurso-. Los contenidos políticos dominan la agenda mediática y existe vinculación partidista en las cabeceras de referencia e influencia del Gobierno en la línea editorial de los medios públicos.

En coyunturas de gran intensidad política y económica, tradicionalmente los partidos multiplican su actividad informativa con el objetivo de movilizar a los ciudadanos afines y, al mismo tiempo, desincentivar la participación de los opuestos (González y Novo, 2011). Simultáneamente, la mayor demanda de datos e interpretaciones por parte de los lectores es el campo adecuado para que los medios intensifiquen su tarea informativa. Se crea, de esta manera, un ciclo perfecto en el que el sistema político, el periodístico y la opinión pública propician un flujo intenso de noticias sobre la situación del país y, con ello, un fortalecimiento de las dinámicas de polarización.

La polarización, por tanto, requiere de la confluencia de los actores políticos y mediáticos para determinar, en fondo y forma, un diálogo tenso que no busca el intercambio de ideas sino la confrontación. En el caso español, la literatura científica coincide que se trata de una característica cíclica que ha determinado el intercambio de mensajes políticos desde los años noventa. Ha de contextualizarse en un mapa político con un parlamento en el que ha imperado el bipartidismo, sin excluir la presencia de fuerzas minoritarias que han tenido influencia en periodos de mayorías simples. Aunque se ha atravesado periodos de menor intensidad, la crispación del debate público es una seña identitaria en España y, por ello, se hace necesario una definición precisa de sus características y actores protagonistas. Más aún, en tanto actualmente se atisba un horizonte incierto de algunas particularidades que, hasta la fecha, se había mostrado inherentes a la polarización y han evolucionado sustancialmente.

\section{Marco teórico. Conceptualizando la polarización en el escenario político y mediático español}

Desde la Ciencia Política se viene estudiando la presencia de la polarización política en diferentes realidades nacionales, especialmente latinoamericanas y la estadounidense, desde los años noventa. Así Corrales ha estudiado cuál es la finalidad de tal práctica, que alcanzó notables cotas en la Venezuela del presidente Chávez (2005:115), y afirma: "La consecuencia de la polarización es, pues, el fortalecimiento de los polos del espectro político y la desarticulación del centro". La definición de 
Corrales se centra en las dinámicas de polarización que se viven dentro del espacio de debate político por los actores que forman parte de él. Aunque su trabajo se circunscribe a la democracia venezolana, con diferencias estructurales insoslayables con la española, su texto proporciona una explicación interesante de cómo se desarrollan estas dinámicas en el espacio público de debate y cómo gobierno y oposición, en el ejercicio de sus roles, son responsables del aumento de la misma.

Un contexto más homologable al español podría apreciarse en los trabajos producidos en Estados Unidos (Baum \& Groeling, 2008; Nivola \& Brady, 2006;).No es hasta hace pocos años, tras el punto de inflexión que supusieron en la opinión pública y la política estadounidenses los ataques del 11S y la guerra contra Iraq en el segundo mandato de George W. Bush, que se aprecia un incremento tangible de la tensión en el país. Fiorina y Abrams (2008), aunque fijan el inicio de la situación unos años antes, afirman que la repetición de los resultados electorales de 2000 en 2004 fue la señal de alarma sobre la distribución bipolar del voto en los estados americanos. Esta situación hizo que se revitalizara el estudio de la polarización en aplicada a países con democracias sólidas; se entendía la polarización no como desestabilizadora económica ni socialmente sino como la forma en la que se estaba realizando el debate público: constantemente imbuida en un tono crispado que buscaba el disenso y el distanciamiento en lugar de crear espacios de consenso. En un país presidencialista y un sistema bipartidista, la situación de la opinión pública en torno a dos posturas parece imperativa, pero se había producido una separación creciente y una tensión entre las mismas sin precedentes.

A partir de todo ello, la polarización puede definirse como el proceso por el que los actores del debate político se posicionan fuertemente en uno de los polos o extremos, alejándose del centro, consiguiendo que esa atracción entre afines suponga una repelencia entre opuestos e intentado movilizar, con ellos, a la opinión pública y a las agendas ciudadanas y mediáticas. Es un proceso activo en el que los actores se mueven, abandonando el centro y se ubican en discursos menos moderados intentando, a su vez, desarticular las posiciones intermedias en el debate público. No es, por tanto, una dinámica natural, sino inducida por fuerzas enfrentadas que buscan atraer a los demás agentes y alejarlos del contrario.

Por tanto, el primer objetivo de este trabajo es definir las características específicas de la polarización española desde los años noventa y analizar el rol desempeñado por los actores políticos y mediáticos en ella. El presente trabajo estudia la importancia de la prensa como agente determinante de este fenómeno y no como resultado del mismo. Maihold (2007) se pregunta cuál es el origen de esta situación, si son los políticos y los medios los que la incentivan o si, al contrario, es un signo de cambio de la propia sociedad española que han interiorizado los políticos. Concluye que la polarización es un concepto relacional, es el tenso resultado de la confrontación de fuerzas opuestas, y que es propiciada desde la vida política. Para este autor, la situación en España es de una crispación general que desarticula el diálogo y es inmoderada tanto en la forma como en el fondo. Entiende, asimismo, por crispación el constante ruido o perturbación del debate público que es alimentado por actores mediáticos, políticos 
o sociales para impedir que la vida pública se desarrolle con normalidad, aun dentro de la confrontación partidista. Es decir, se sobrepasan los límites de la necesaria confrontación para caer en el ruido constante que eclipsa a ésta y sólo permite un diálogo tenso e infructuoso

La bibliografía científica sobre la polarización en España señala unánimemente que las legislaturas 1993-1996 y 2004-2008 son las que más elementos de crispación concitaron (Field, 2009; Canel \& Sanders, 2006; Balfour, 2005). Así, entre otros, el Informe sobre la democracia en España 2008. Derrota, pero no fracaso explicaba que el precedente de la crispación de la legislatura 2004-2008 se halla en la de 19931996. Se sostiene que en ambos periodos el PP usó la estrategia de la crispación como principal eje político: "El PP utilizó todas las armas políticas y parapolíticas ante la imposibilidad de vencer en las urnas: en 1993, en medio de una aguda recesión económica, con tres devaluaciones seguidas de la peseta, la emergencia de los primeros escándalos de corrupción en las filas de los socialistas y tras once años de gobierno ininterrumpido, Felipe González vuelve a ganar las elecciones" (2009: 19). Si bien no se ignoran los elementos del discurso polarizado que estuvieron presentes en las dos legislaturas gobernadas por José María Aznar (Barrera, 2005; Maihold, 2007). Por ello, este artículo se centra en este marco temporal para trazar las características endémicas de la polarización del sistema español de medios y, a su vez, reseñar las diferencias.

Los términos en que se desarrolla esta crispación se definen por la desmesura en la crítica al adversario que dejó paso a la descalificación sistemática. El discurso político y periodístico durante estas legislaturas se desempeñó con constante crispación, un debate inmoderado en las formas y agresivo en el fondo con la finalidad de polarizar a la opinión pública y obtener titulares gruesos (Cotarelo 1995). Bezunartea, Cantalapiedra y Del Hoyo (2000), tras analizar el tratamiento de la política durante 1993-1996 en El Mundo, Abc y El País, afirmaron que la opinión invadió los espacios informativos, que se sobredimensionaron los acontecimientos y que se permitió que los hechos se tergiversaran y distorsionaran al servicio de determinado objetivos. La polarización persigue la creación de tensión, para obtener réditos electorales, antes que el debate e intercambio de ideas políticas.

Se parte, por tanto, de la hipótesis (H1) de que el protagonismo de la prensa escrita en las dinámicas de crispación vividas en España en estos años fue determinante, a pesar de que se trata de un país en el que tradicionalmente los índices de lectura de prensa son bajos. Resulta importante determinar el rol adquirido por los medios de comunicación en estos periodos y estudiar las analogías y divergencias entre dichas legislaturas y, especialmente, entre el papel desempeñado por la prensa de referencia.

La polarización española tiene su manifestación más evidente en el crecimiento sostenido del bipartidismo. Así, en las elecciones generales de 1993 PSOE y PP sumaban el $73,54 \%$ de los votos y esta cifra ha ido creciendo constantemente hasta los comicios de 2008, cuando acapararon el $83,81 \%$ de los sufragios. Sin embargo, en 2011, a pesar de la abrumadora mayoría absoluta del PP, el bipartidismo cayó 
hasta el $73,35 \%$. Esta es la primera señal que alarma sobre el posible cambio del escenario de futuro de la polarización española e, indudablemente, si dicha evolución se produce, tendrán consecuencias tangibles para el sistema mediático. Por ello, el segundo objetivo de este trabajo es trazar las características que apuntan hacia un discurso político y mediático que supere la bipolarización antagónica que comienza a gestarse en la presente legislatura (Sampedro y Seoane, 2008). En el actual escenario de crisis económica, se sostienen las hipótesis (H2) de que la prensa escrita se está reubicando ideológicamente, lo que ha hecho evolucionar el rol que desempeñan en las dinámicas de crispación, y de que han surgido nuevos actores, mediáticos y políticos que están desempeñando un papel determinante en el tenso debate público, llegando a un público masivo a través de la televisión (H3).

\section{Metodología}

Este trabajo combina el estudio y contextualización histórica de los periodos analizados, en términos mediáticos y políticos, con el análisis de contenidos de prensa y televisión. Se trata de un trabajo descriptivo y crítico y, por ello, la metodología se basa en la triangulación de técnicas cualitativas, historiográficas y cuantitativas.

Así pues, en primer lugar, la metodología comprende la revisión de la literatura científica sobre la historia política reciente de España. En segundo lugar, para completar el estudio del contexto político, se ha recurrido a fuentes primarias como los barómetros del CIS, los datos del Estudio General de Medios, los Anuarios de la Prensa y los resultados electorales de los periodos analizados (1993-1996, 20042008, 2011-2015).

En tercer lugar, se ha llevado a cabo un extenso análisis de contenido de la prensa escrita durante los periodos analizados y, en la última legislatura, se ha complementado con el estudio de los contenidos televisivos. Así, durante los primeros periodos se ha analizado los contenidos de El Mundo, Abc y El País, en profundidad, y se ha realizado un estudio de caso de la cobertura de determinados asuntos especialmente significativos: casos de corrupción en los años noventa y asuntos de política social en la primera legislatura de José Luis Rodríguez Zapatero, fundamentalmente. Para el último mandato, se ha incorporado el análisis de La Razón y los contenidos políticos de las cuatro cadenas privadas de televisión con más audiencia -Telecinco, Antena 3, laSexta y Cuatro-.

Existe un amplio campo de investigación en el que historia política y mediática españolas confluyen y en él se sitúa el presente estudio. Por ello, la metodología requiere de la conjugación multidisciplinaria de las técnicas cualitativas y cuantitativas en su análisis. 


\section{Análisis: Características endémicas de la crispación mediática española desde los años noventa hasta 2008}

Hay una serie de características que se han mantenido inalterables y han sido decisivas en las dinámicas de crispación que ha caracterizado a la prensa y la política españolas desde los años noventa. Con respecto a las del ámbito político, la primera que cabe señalar es el signo del partido que gobernaba. En ambos casos, presidía el PSOE con mayoría simple, lo que obligó a pactos -puntuales o de gobierno- en los dos periodos. Son minoritarios los análisis que apoyan que la segunda etapa de polarización se inició durante el gobierno de Aznar. En el caso español, la crispación es una estrategia discusiva alimentada por los partidos en la oposición para magnificar el alcance su tarea y desgastar al Ejecutivo; por lo tanto, en ambos mandatos, proviene del mismo actor político.

Con respecto a las características mediáticas, la principal es el protagonismo de la prensa escrita- lo que ya es, per se, relevante pues es éste en un país con bajos índices de lectura de prensa-, si bien es necesario matizar que, según la legislatura, ésta ejerció un rol diferenciado. Bajo el gobierno de Felipe González la prensa, capitaneada por El Mundo, fue crucial para sacar a la luz escándalos que, de otra forma, no hubiera conocido la opinión pública. Sin las investigaciones periodísticas y filtraciones no se habría llegado a juzgar el caso de los fondos reservados del Ministerio de Interior o del patrimonio ilícitamente acumulado por el director de la Guardia Civil, Luis Roldán, por ejemplo. Junto a estos casos, en los que la interpretación del medio no coincidió totalmente con las sentencias judiciales pero sí hubo condenas de altos cargos nombrados por el Gobierno, se publicaron otros muchos que se olvidaron en cuanto las declaraciones de los protagonistas venían a desmentirlos. La última legislatura de Felipe Gónzalez llegó precedida por la dimisión del vicepresidente Alfonso Guerra por las irregularidades cometidas por su hermano, el inicio de la investigación del caso GAL o la instrucción del caso Filesa, como asuntos destacados. Para hacerse una idea de la importancia de la prensa, estos casos habían sido publicados o ampliamente difundidos por Diario 16, primero, -Juan Guerra- o El Mundo, -Filesadespués.

En el gobierno de Zapatero, sin embargo, al no tratarse de escándalos sino de interpretaciones polarizadas de la realidad política y de la acción de Gobierno, los periódicos fueron fundamentales al avivar los argumentos más crispados frente a las decisiones del Ejecutivo y seleccionar los testimonios más infamantes (Thompson, 2000). El debate crispado no estuvo vinculado a escándalos sino a la gestión política. En el caso de El Mundo hay que destacar su labor investigadora para mantener durante años la teoría de la conspiración sobre los atentados del 11M (Sampedro, 2008). En 2004, los medios y los populares realizaron una dura oposición en torno a este tema, el debate sobre el Estatuto de Cataluña, las leyes sociales y el diálogo con ETA, fundamentalmente (Rodríguez- Castromil, y Rodríguez Díaz, 2011). A pesar de ello, las dos legislaturas se desarrollaron en términos similares: consideración del gobierno como deslegitimado, ataque a los valores fundamentales del Estado como 
1a Transición o la Constitución y discurso infamante. La televisión, por el contrario, el medio más consumido por los españoles, se situó en un tono más neutro sin perder audiencia.

El discurso político y periodístico estuvo caracterizado por una inmoderación en las formas, una tensión en el debate que no buscaba la confrontación de ideas sino la polarización de la opinión pública. Se entrelazaron información y opinión en las páginas de la prensa pues parte de ésta decidió adquirir un protagonismo singular. Como muestra de ello, cabe citar que en 1994 algunos de los más conocidos periodistas y directores críticos con el Gobierno de González se unieron en la AEPI (Asociación de Escritores y Periodistas Independientes) y publicaron sus principios fundacionales en los que desgranaban los motivos de su oposición a los socialistas y la actitud beligerante que habían decidido adoptar (los directores de El Mundo, Abc y Diario 16, Luis de Olmo, Federico Jiménez Losantos, Antonio Herrero, José María García...).

Se permitió un acceso desigual de las fuentes políticas a los diferentes medios y proliferó la publicación de "no acontecimientos" o indicios de escándalos que se incrementaban en el medio y que deshacían súbitamente sin ofrecer explicaciones al lector cuando no coincidían con la línea interpretativa sostenida (Teruel, 2006). Igualmente, Canel y Sanders (2005 y 2006) afirman que, en la cobertura de los escándalos, la prensa española tendía hacia una polarización de marcado carácter político, estableciendo 'un nosotros' -críticos de los socialistas y sus malas acciones- y un 'ellos' -los socialistas y sus malas acciones-. En esta cobertura, el enfoque o marco discursivo que los medios transmitieron tuvo un extraordinario poder creando conceptos y "encapsulando" valores que han pervivido en el tiempo y se han convertido en elementos emblemáticos de dicho momentos histórico. La cobertura de escándalos o hechos críticos fueron momentos claves para que los partidos y periódicos intentaran imponer dinámicas polarizadoras que indujeran a un sobredimensionamiento de los escándalos e impusieron una retórica infamante (Teruel, 2013). Se crea un discurso en el que los escándalos se entrelazan creando un clima constante de alteración de la vida política que se quiere hacer ver como inherente a la democracia.

Así pues, a colación de estas dos características, los periódicos se convierten en los escenarios recurrentes donde la oposición se expresa con un discurso agresivo sobre estos temas. Si bien, el aumento del consumo de medios fue generalizado pues la audiencia de las radios informativas, especialmente de las tertulias matutinas, se incrementó también en estos años y destacaron figuras como Iñaki Gabilondo en Hoy por hoy (Cadena Ser) y Federico Jiménez Losantos en La Mañana (Cope). Se deslocaliza la crítica al Gobierno, que se trasladó del Congreso y el Senado hacia los medios de comunicación de forma que se buscaba más el eco mediático que el verdadero intercambio de opiniones.

Los dos actores enunciados - la oposición y parte de los medios- consideran que el acceso del PSOE al poder fue ilegítimo; ésta es otra característica determinante de estos periodos. Se reafirma de esta manera lo que Corrales asevera al respecto: que el inicio de las dinámicas de polarización se asocia con el 'asalto al poder' real 
o percibido como tal por otros actores. En el primer periodo, los sondeos previos vaticinaban una victoria del PP que no llegó a producirse. Los populares y los medios afines iniciaron una campaña de deslegitimación del Gobierno bajo los argumentos de que el voto del PSOE era un voto cautivo por las subvenciones y subsidios que recibían las clases más necesitadas o los afines al partido y por la manipulación de la información que se ofrecía desde los medios públicos. Por tanto, la victoria en 1993 se consideró inesperada y pronto comenzó a ser cuestionada su legitimidad desde la oposición que, ya en 1994, hizo célebre la famosa exhortación ¡Váyase, señor González!, entre otras muchas consignas que abonaban su tesis de la que ellos denominaron como "necesidad de regeneración democrática".

En 2004, los atentados del 11M en Madrid y la investigación sobre su autoría han sido un motivo de cuestionamiento sobre la legitimidad de la victoria socialista durante años (Sampedro 2008). Tres días antes de los comicios de marzo, a los cuales concurrían José Luis Rodríguez Zapatero (PSOE) y Mariano Rajoy (PP), tuvieron lugar los ataques terroristas. Este acontecimiento clave en la política española se convirtió en un momento determinante para intentar controlar la opinión pública a favor de autorías antagónicas. Se confrontaron dos universos simbólicos opuestos; por una parte, el del Gobierno que atribuía la autoría a ETA e instaba a la cohesión ciudadana bajo los postulados del PP. Entre estos, uno de los más destacados fue la defensa a la Constitución y al centralismo como argumento transideológico con el que intentaron reagrupar al voto. Por el otro, la pista cada vez más incuestionable de Al Qaeda, a la que el resto de partidos fue paulatinamente dando credibilidad, que penalizaba al PP por sus políticas anteriores -participación en la guerra de Iraq- y por su gestión informativa de los atentados. La victoria de Zapatero estuvo, pues, precedida por esta catástrofe y, para el PP y ciertos medios, ello la convierte en un mandato sin legitimidad. En palabras de Balfour: "Insisten en la ilegalidad del resultado de marzo 2004, ilegalidad no sólo porque el PSOE supuestamente instrumentalizó el atentado de Madrid del 11M para fines electorales sino también porque se han ocultado indicios que podrían probar que ETA estuvo detrás del atentado" (2007: 384).

Atendiendo al primer objetivo planteado para este trabajo -definir las características de la polarización y el papel desempeñado por los actores políticos y mediáticos- cabe concluir que la polarización del discurso político y mediático españoles se asocia a un Gobierno del PSOE en mayoría simple, una victoria inesperada que fuera conceptualizada como ilegítima por la prensa y la oposición y un elevado protagonismo y consumo de la prensa escrita. Estas tres variables, resumidamente, han sido imprescindibles para la existencia de un debate público crispado en el que las formas se volvieron inmoderadas y el ruido alimentado por actores mediáticos, políticos o sociales saturó la actualidad informativa para impedir que la vida política se desarrollara con normalidad.

A pesar de las notables coincidencias expresadas, hay variables que plantean sustanciales diferencias entre los escenarios de crispación vividos en ambos periodos. Existen una serie de características que no han sido tan determinantes como se estimaba y que han marcado las diferencias entre ambas legislaturas. Así, Balfour, 
aunque encuentra conexiones entre ambas legislaturas, analiza las diferencias: "No creo que ésta responda simplemente a una reedición aún más vehemente de su estrategia de acoso populista desde la oposición del periodo 1993-1996, cuando el gobierno de Felipe González estaba en minoría como lo está el actual gobierno de José Luis Rodríguez Zapatero. Son dos periodos muy diferentes. En el 93-96, el PSOE estaba al final de un largo ciclo de poder, sufría una serie de escándalos y había perdido el apoyo de su base electoral, lo que se había expresado en una creciente abstención" (2007: 385).

Con respecto al ámbito político, las principales diferencias estuvieron en la cohesión interna y la renovación de los dos partidos mayoritarios. Se mantuvo un congreso bipartidista pero Gobierno y oposición llegaron de forma muy diferente a cada uno de estos periodos. En 1993, el PSOE evidenciaba tensiones internas e incapacidad de renovación tras tres mandatos consecutivos -los dos primeros con mayoría absoluta-. El goteo de escándalos que les rodeaba había erosionado la imagen de Felipe González, aunque seguía siendo el político más valorado (Méndez-Lago, 2005). En 2004, sin embargo, tras ocho años de peregrinación por el desierto y resurgimiento, el partido, liderado por Rodríguez Zapatero, tenía una ejecutiva nueva y se mostraba cohesionado. El PP, por su parte, siguió una dinámica casi opuesta pues, en 1993, acudió a las urnas fortalecido y unido en torno a la figura de José María Aznar y, en 2004, se inició una regeneración interna de la mano del sucesor que éste nombra, Mariano Rajoy.

En el ámbito mediático, aunque el tono de debate crispado se mantuvo en similares niveles de intensidad, éste se refería, entre 1993 y 1996, a la corrupción y la mala situación económica y, en 2004- 2008, a asuntos de estado y decisiones en materia de derechos sociales que adoptó el ejecutivo. En este último periodo, el discurso crispado se organizó en torno a tres temas en concreto -el 11M, el Estatuto de Cataluña $y$ el alto al fuego de ETA- $y$, en menor medida, en torno a leyes como el matrimonio homosexual (Estefanía, 2009). Ello verifica la tesis sobre la importancia de la labor de los medios en la polarización vivida en España por su capacidad para introducir temas en el debate público. Así, por ejemplo, cuando el líder popular Mariano Rajoy acusó al presidente Zapatero de "haber traicionado a los muertos de ETA" por dialogar con la banda terrorista, junto con el rechazo de la opinión pública y gran parte de clase política, encontró el apoyo de su partido y de periódicos como $A b c$ (Teruel, 2013).

Las dos últimas diferencias reseñables entre ambos periodos son la situación económica -pues, tras la crisis de los noventa, en 2004 España se encontraba en pleno crecimiento económico- y el nacimiento de las redes sociales. En estos años empieza a asomar la importancia de las redes sociales para la movilización de la opinión pública. En la primera de las legislaturas analizada las tecnologías de la comunicación no permitían un fenómeno como el que se vivió en 2004 y, posteriormente, en otros acontecimientos como el 15M (Sampedro, 2005). Pero el 11M fue el primer acontecimiento en el que estas tecnologías propiciaron una movilización de la opinión pública de forma viral. 


\section{La polarización en el escenario de crisis política y mediática a partir de 2011}

El análisis histórico del fenómeno de la polarización no deja lugar a dudas: es una característica definitoria del sistema español de medios y, aunque ha tenido periodos de menor intensidad, nunca ha desaparecido por completo. Algunas de las variables que influyen en este fenómeno son el signo del gobierno con mayoría simple tras una victoria inesperada, el tono de la oposición y el papel protagonista que juega la prensa. En ambas legislaturas se observó un discurso político y mediático en términos de constante crispación e inmoderación en las formas. Y, por otra parte, puede afirmarse que la polarización es independiente de la situación interna de los partidos, porque lo determinante es el liderazgo que desarrolla la prensa -como se planteaba como hipótesis de este trabajo-, y que puede surgir en torno a escándalos concretos o a asuntos de estado y cuestiones ideológicas derivadas de la acción de Gobierno.

Sin embargo, en el momento actual concurren una serie de factores radicalmente distintos de los enunciados en los epígrafes anteriores y que ofrecen argumentos antagónicos sobre la pervivencia de la crispación en los mismos términos. Es decir, hay nuevas variables que van desde las netamente politológicas hasta las mediáticas y que apuntan en sentidos contradictorios sobre la dosis de polarización que soporta el debate público español actualmente. Por ello, sin ignorar la dificultad de analizar el escenario político y mediático en la recientemente concluida legislatura (desde 2011), se hace necesario señalar las posibles características que apuntan hacia una ruptura del mapa de polarización española en los términos tradicionales y la presencia de nuevos elementos.

La diferencia política más evidente de este periodo con respecto a los anteriores es el signo del gobierno: preside el PP con mayoría absoluta. Nos hallamos inmersos en una grave crisis económica y política. Aunque el paro es la mayor preocupación para los españoles, la frágil confianza en la política de la mayoría de los ciudadanos ha elevado, según los barómetros del CIS, la preocupación por la corrupción $(63.8 \%$ en noviembre de 2014) y los partidos políticos (31.4\% en marzo de 2013) a las cifras más altas alcanzadas desde que existen registros (Llaneras, 2015). De acuerdo con estos datos sociológicos, la legislatura 2011-2015 guarda más similitudes con la de 1993-1996 que con la de 2004-2008 pues se registran valores medios similares de preocupación por la corrupción: 27.6 como valor medio durante toda la legislatura de los noventa y 39.5 en la más reciente, frente al 0.7 como valor medio entre 20042008.

Otra diferencia significativa abordada desde la Sociología es la movilización ciudadana y la participación política alcanzada en esta última legislatura. Frente a quienes sostienen que el clima de negativismo mediático puede desincentivar la participación ciudadana, se viene afirmando que la abundancia de información redunda en la creación de una ciudadanía más instruida en materia política y mediática para la cual los casos negativos o escándalos no suponen un motivo de desánimo de su interés en política sino, al contrario, un motivo para demandar mayor información (Norris, 
2000). Se trata del círculo virtuoso: la información negativa refuerza, a largo plazo, el activismo y compromiso de los ciudadanos que demandan una mayor información

Así pues, desde 2011, se ha producido una repolitización del espacio público sin precedentes. Esto es, la sociedad civil se ha organizado en numerosos movimientos para actuar contra la acción política: Marea Blanca, Marea Verde, 15M, Stop Desahucios... (Rodríguez-Polo, 2013; Martínez Nicolás, 2011). Se ha confirmado la hipótesis de que el aumento de los niveles educativos y las habilidades y recursos de que disponen los ciudadanos, en un contexto de crisis económica, han espoleado la participación social (Morales, 2005).

Por tanto, la polarización crea interés por la información, moviliza a los ciudadanos, es aprovechada por las empresa mediáticas para atraer lectores (Bernhardt, Krasa y Polborn, 2006) y no ha supuesto en España una pérdida de credibilidad en los medios en cuanto se mantiene la tendencia a consumir prensa que no ocasione una disonancia cognitiva (Fraile y Lewis-Beck, 2010). Así pues, la sociedad española está movilizada políticamente y consume más información política. Todo ello ha conllevado que el medio masivo por excelencia, la televisión, haya multiplicado los contenidos políticos en su parrilla y haya adquirido un protagonismo destacado al añadir nuevos actores partidistas al debate público (Humanes et al., 2013).

Gráfico 1. Horas diarias dedicadas a política en televisión privada generalista

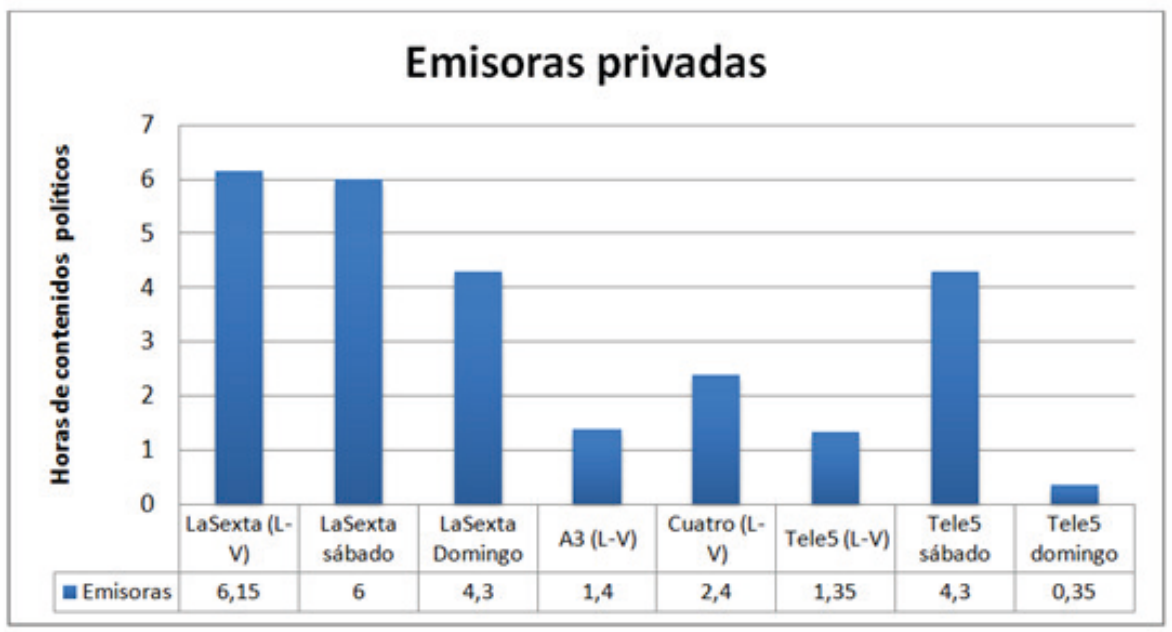

Fuente: elaboración propia

Han proliferado los programas de tertulia política en las cadenas privadas generalistas. Junto a los informativos, en los que se ha separado el espacio de deportes para centrarse en política nacional e internacional, estas emisoras ofrecen tertulias en las que cabe hablar de pluralismo polarizado interno pues las componen contertulios- 
tanto periodistas como políticos- con puntos de vista fuertemente enfrentados que buscan el intercambio de ideas fuerza más que argumentos constructivos.

LaSexta dedica más de seis horas diarias de su programación, tanto entre semana como los sábados, a contenidos políticos en información o tertulia ${ }^{1}$ (gráfico 1). Con más de un $32 \%$ de su tiempo de emisión diario es la cadena que más contenidos políticos ofrece frente a Antena 3, que se queda en el 8,3\%. Las dos emisoras de Atresmedia marcan los dos extremos en tiempo de emisión sobre política y, a su vez, representan líneas editoriales opuestas. En 2011, en el estudio del Anuario de la Profesión Periodística sobre la percepción de los espectadores, LaSexta era la cadena nacional que se ubicaba más a la izquierda y Antena 3, a la derecha ${ }^{2}$. La estrategia empresarial tras la fusión-absorción de ambas cadenas ha pasado por mantener líneas editoriales diferencias que permitan multiplicar su target.

Su éxito comercial radica en programas como Salvados, que duplican la audiencia media de la cadena: frente al 7,2\% que obtuvo la emisora, el programa de Jordi Évole llega al $15,2 \%$ de media en $2014^{3}$. Junto a este espacio, otros buques insignia de la programación son El Intermedio, con un 13,7\% de media, y LaSexta Noche, que ha llegado a alcanzar al 15,9\% de la audiencia.

De esta manera, el tándem formado por las cadenas de Atresmedia presenta dos perfiles muy diferenciados de programación con un protagonismo de la política radialmente distinto. En Mediaset, por su parte, Telecinco y Cuatro los contenidos políticos ocupan un porcentaje más parecido de la parrilla $-9,9 \%$ de media en la primera y $2,2 \%$ en la segunda de lunes a sábado- pero en horario y días diferentes para complementar la oferta.

El éxito de los espacios de tertulia política ha ido paralelo al nacimiento del partido Podemos y, posteriormente, de Ciudadanos; tanto es así, que desde los partidos mayoritarios se achaca el éxito de esta nueva formación al espacio concedido a sus líderes en las cadenas privadas. Mientras que el surgimiento de UPyD, en 2007, tuvo una cobertura mínima por parte de la prensa generalista, traducida en 62 editoriales y columnas en un año desde la fecha de su fundación en 2007, el partido de Pablo Iglesias ha alcanzado las 341 menciones en la sección de opinión en los cuatro periódicos nacionales en el último año: El País, El Mundo, Abc y La Razón (Teruel, 2014). Y es que todos los sondeos electorales parecen confirmar el éxito de las nuevas formaciones que aspiran a desdibujar el bipartidismo español y que, por otra parte, favorece la polarización político mediática (Llera, 2013). En España, desde los años noventa, populares y socialistas alcanzan en torno al $80 \%$ de los escaños del Parlamento -de hecho, en 2008 coparon el $83.81 \%$ de los sufragios- pero, según el sondeo de intención de voto del CIS para febrero de 2015, esta tendencia está en declive pues ambos se quedarían en un $51.2 \%$. Este barómetro del CIS da una estimación de voto a PP de $27,3 \%$, PSOE $22 \%$, Podemos 23,9\%, IU 5,2\% y UPyD 4,6\%: todos estos grupos serían necesarios para acaparar hipotéticamente tantos escaños como populares y socialistas alcanzaron en 2008. En las elecciones del 20 de diciembre el bipartidismo 
alcanzó el peor resultado de su historia por el surgimiento de nuevas fuerzas: PP 123 escaños, PSOE 90, Podemos 69 y Ciudadanos 40.

Así pues, partiendo de una sociedad movilizada políticamente y de un incipiente escenario de quiebra del bipartidismo, la polarización mediática también se redibuja. Con respecto al escenario mediático, se observa el posicionamiento muy claro en medios de derecha - La Razón y Abc- e izquierda - LaSexta, Cuatro-, pero se ha modificado el rol de la prensa más leída. El Mundo, que por su estilo ha sido protagonista de las dinámicas de crispación, se ha distanciado de la gestión de Rajoy a raíz de los casos de corrupción que rodean al PP y por críticas a su gestión. Este distanciamiento, que tuvo su corolario en la publicación de una exclusiva con Luis Bárcenas -ex tesorero del PP- en que se acusaba a destacados miembros del partido de cobrar sobresueldos ilegales se saldó, según su protagonista, con la destitución del director del diario desde su fundación, Pedro J. Ramírez.

En el ámbito de los periódicos, por ello, se hace necesaria una investigación diacrónica pues todo parece indicar que, por una parte, la acostumbrada alineación de la prensa se ha reforzado en los extremos pero se han atenuado los lazos partidistas de los diarios más leídos. El Mundo y El País se han alejado de los partidos a los que tradicionalmente eran afines: el primero de ellos a raíz del caso Gürtel y, el segundo, tras episodios como la llamada "guerra del fútbol" y tras el nombramiento de su nuevo director, que declaró que el periódico no era afín al $\mathrm{PSOE}^{4}$ (Barrera, 2005; De Bustos y Pozas, 2009).

Por otra parte, el asentamiento de diarios informativos digitales y su crecimiento progresivo en audiencia es otra característica definitoria del sistema de medios actual que requiere un estudio de su evolución. Frente al estancamiento de ventas de la prensa escrita y su crisis empresarial, materializada en sucesivos EREs en todas la cabeceras, estas nuevas empresas mediáticas está en crecimiento constante. Han proliferado medios digitales de tendencia progresista que desarrollan una labor de investigación constante sobre los escándalos políticos: eldiario.es, Público, Infolibre... Así, por ejemplo, eldiario.es ha alcanzado los 5.381.087 lectores únicos en diciembre de 2014 -creciendo un millón y medio en dicho año-. Estos medios se sitúan en un entorno virtual en el que las redes sociales dibujan un escenario de relaciones políticas polarizadas (Bustos y Capilla, 2014; Aragón et al, 2013).

\section{Conclusiones}

El análisis de las características de la polarización político y mediática de la presente legislatura viene a plantear la continuidad de sus manifestaciones más sustanciales en el espacio de debate público pero, también, que se hallan en un punto de ruptura algunas de las variables que han sido definitorias de los periodos anteriores. Aunque prevalece el estilo de debate tenso, inmoderado en las formas y sin finalidad constructiva y la balcanización que caracteriza a los enfrentamientos entre los actores 
del espacio público hay diferencias medulares: la existencia de un gobierno del PP con mayoría absoluta supone una primera divergencia fundamental. Junto a ello, el horizonte de quiebra del bipartidismo, la reubicación de los periódicos en el espectro partidista - con algunos más polarizados en los extremos y la prensa de referencia con un posicionamiento más oscilante- y el protagonismo de la televisión privada marcan un planteamiento sustancialmente diferente.

En el ámbito de los medios, el periodismo escrito busca su nuevo rol en un mercado de la prensa en crisis y con la oferta mediática digital creciente, como sostenía la segunda hipótesis de este trabajo. La influencia de la prensa escrita sigue siendo elevada para fijar la agenda política y destapar escándalos como la trama Gürtel o el caso Bárcenas, ambos con exclusivas de El País y El Mundo; sin embargo, se registra una caída imparable de sus ventas. Entre febrero de 2015 y 2015, El Mundo y El País se han dejado un $13 \%$ y $11 \%$ de sus ventas respectivamente según EGM; sólo el diario de Prisa sobrepasa los 110.000 ejemplares diarios. Aunque existen datos que demuestran que se ha producido un alejamiento partidista de los grandes diarios con respecto a las dos formaciones mayoritarias, se observa, paralelamente, una defensa de la estabilidad que tradicionalmente ha traído bipartidismo a España frente a la inseguridad, entienden, que supondría el fortalecimiento de nuevas formaciones. Pues, si bien, en un primer momento, muchos de estos nuevos partidos -UpyD, Equo, Vox- fueron recibidos con satisfacción por los medios en tanto parecía que no podían alterar el reparto de poder (Teruel, 2014), a medida que Podemos ganaba en intención de voto empezaron a arreciar las críticas desde sus páginas a la desestabilización que conllevaría en el mapa político5.

El protagonismo adquirido por la televisión como generadora de contenidos políticamente polarizados es una novedad sustancial. El auge de la tertulia política en los medios privados la convierte en referente de los públicos masivos en los argumentos polarizados. Tanto es así, que los partidos han renovado sus cabezas de cartel respaldando el éxito de este formato: Antonio Carmona -candidato del PSOE a la alcaldía de Madrid- y Tania Sánchez -candidata (ahora dimitida) de IU a la Comunidad de Madrid- son contertulios fijos en "LaSexta Noche" y Pablo Iglesias lo ha sido durante meses en "Las mañanas de Cuatro", por ejemplo-. Es esta cadena de Atresmedia, visiblemente situada a la izquierda, la que mejores réditos ha sacado a los contenidos políticos pues ha marcado, entre 2014 y 2015 , las mejores cifras de audiencia en su década de vida.

Por todo ello, la polarización político-mediática española requiere un análisis de su evolución. No cabe duda de que el debate público se encuentra crispado pero no es menos cierto que ningún periodo de polarización ha durado en España más de una legislatura. En el caso de Zapatero, la segunda legislatura rebajó sustancialmente el tono de la oposición y, con Felipe González, tras 1996 hubo un cambio de ciclo con el gobierno de Aznar. Tanto si revalida su triunfo el PP, como si se produce un gobierno de izquierdas -de manos del PSOE junto con Podemos o Ciudadanos- se prevé un escenario de gobierno muy diferente al actual y, a tenor de las tendencias que viene recogiendo el CIS, será un gobierno que requiera pactos y negociación. Así pues, a 
partir de 2016 podrá estudiarse cómo evoluciona este estilo discusivo de los medios que puede entenderse, tanto en el caso de la prensa escrita como la televisión, como una estrategia empresarial de posicionamiento en el mercado en tiempo de crisis.

La televisión privada, que se ha multiplicado sus espacios de tertulia política tensa y urgente, se ha convertido en un referente ideológico para la ciudadanía aunque es una estrategia que, a largo plazo, puede saturar al espectador y hacer que pierda credibilidad. Del mismo modo, la prensa escrita intenta sobrevivir al escenario de crisis económica renovando sus contenidos, siendo más permeable a las nuevas tecnologías y evidenciando una reubicación ideológica ante el nuevo escenario multipartidista. Todo ello, junto a la pujanza de los medios digitales y la mayor demanda informativa de una sociedad interconectada, movilizada y activa a través de las redes sociales y con una gran demanda de contenidos informativos, plantea un horizonte mediático en España que requiere de un análisis continuo sobre la evolución de las dinámicas de polarización.

\section{Referencias bibliográficas}

BALFOUR, S. (2005).'The reinvention of Spanish conservatism: the Popular Party since 1989". In The Politics of Contemporary Spain, BALFOUR S. (ed). Londres/ Nueva York: Routledge.

BALFOUR, S, y QUIROGA, A. (2007). The reinvention of Spain: nation and identity since democracy. Oxford: Oxford University Press.

BARRERA DEL BARRIO, C. (2005). "Los Medios de Comunicación durante los gobiernos de Aznar (1996-2004): Un panorama cambiante." En Sphera publica: revista de ciencias sociales y de la comunicación. Vol. 5. P. 165-180.

BAUM, M. A., \& GROELING, T. (2008). "New media and the polarization of American political discourse". En Political Communication, 25(4), 345-365. DOI: 10.1080/10584600802426965

BERNHARDT, D., KRASA, S. Y POLBORN, M. (2008). "Political polarization and the electoral effects of media bias". En Journal of Public Economics. N ${ }^{\circ} 92$ (5). P. 1092-1104. doi:10.1016/j.jpubeco.2008.01.006

BEZUNARTEA, O.; CANTALAPIEDRA, M.J.; DEL HOYO, M. (2000) "La huella de una conspiración mediática”. En Estudios de Periodística VIII Periodismo como actor político. Facultad de Ciencias Sociales, Universidad de Vigo. Pontevedra: Diputación Provincial de Pontevedra, D.L.

BUSTOS DÍAZ, J.; CAPILLA DEL FRESNO, L. (2013) “Twitter y la polarización del debate político: análisis del caso \#objetivodeguindos y \#aznara3". En Historia y Comunicación Social. Vol. 18 N Especial Noviembre. P. 499-509 doi:10.5209/ rev HICS.2013.v18.44260

BUSTŌS, J. C. M., \& POZAS, V. S. (2009). “¿Polarización ideológica o económica?: relaciones entre los medios y el poder político y corporativo". En Viento sur: por una izquierda alternativa, (103), p. 43-52. 
CANEL CRESPO, M.J.; SANDERS, K. (2005) "El poder de los medios en los escándalos políticos: la fuerza simbólica de la noticia icono" En Anàlisi, 32. P. 163-178

CANEL CRESPO, M.J.; SANDERS, K. (2006) Morality Tales. Political Scandals and Journalism in Britain and Spain in the 1990's. Cresskill: Hampton Press

CORRALES, J. (2005) "In Search of a Theory of Polarization: Lessons from Venezuela, 1999-2005", en Revista Europea de Estudios Latinoamericanos y del Caribe, Vol. 79, p. 105-118.

COTARELO, R. (1995) La conspiración, el golpe de estado difuso. Barcelona: Ediciones B.

ESTEFANÍA, J.( 2009). Informe sobre la Democracia en España 2008. La estrategia de la crispación, derrota pero no fracaso. Madrid: Fundación Alternativas.

FARIAS BATLlE, P. (2012) Informe Anual de la Profesión Periodística 2011. Asociación de la Prensa de Madrid: Madrid

FIELD, B. N. (2005) "De-thawing democracy: the decline of political party collaboration in Spain (1977-2004)". En Comparative Political Studies, vol. 38, no. 9, p. 1079-1103.

FIELD, B. N. (2009). "A 'second transition' in Spain? Policy, institutions and interparty politics under Zapatero (2004-8)". En South European Society and Politics, 14(4), p. 379-397.

FIORINA, M. P., \& ABRAMS, S. J. (2008). "Political polarization in the American public". En Annual Review of Political Science, $n^{\circ}$., 11, p. 563-588.

FRAILE, M.; LEWIS-BECK, M. S. (2010) "Economic voting in Spain: A 2000 panel test". En Electoral Studies, 2010, vol. 29, no 2, p. 210-220.

GONZÁLEZ RODRÍGUEZ, J. y NOVO, A., "The Role of the Media Agenda in a Context of Political Polarization" en: Comunicación y Sociedad, vol. XXIV, n. 2, 2011, pp.78-131.

GURRIONERO, M. Y SANDERS, K. (coords.). Estudios de Comunicación Política. Libro del año 2012. Tecnos: Madrid.

HALlin, D., MANCINI, P. (2003), Comparing Media Systems; Three Models of Media and Politics, Cambridge University Press.

HEYWOOD, P.M. (2005) "Corruption, democracy and governance in contemporary Spain”. En Balfour, S. (ed.) The Politics of Contemporary Spain. (Pp 39-60). Londres/ Nueva York: Routledge.

HOPKIN, J. (2005) "From consensus to competition: the changing nature of democracy in the Spanish transition". En Balfour, S. (ed.) The Politics of Contemporary Spain. (Pp 6-26.) Londres/ Nueva York: Routledge.

LLERA RAMO, F. J. (2013) "Las elecciones de 2011 en España: cambio de ciclo político en un contexto de crisis" En VALENCIA SÁIZ, Á. (coord.) Elecciones en España y Andalucía 2012. Análisis y tendencias de cambio. Centro de Estudios Andaluces, Sevilla.

MAIHOLD, G. (2007) "La crispación. Instrumentos y efectos de la polarización política en España" En BERNECKER, W.L.; MAIHOLD, G. (eds) España: del consenso a la polarización. Cambios en la democracia española. Madrid: Bibliotheca Iberoamericana. P. 393-408. 
MÉNDEZ-LAGO, M. (2005). "The Socialist Party in Government and in Opposition'. En, BALFOUR, S. (2005) The Politics of Contemporary Spain. Londres/ Nueva York: Routledge.

MORALES, L. (2005) “¿Existe una crisis participativa? La evolución de la participación política y el asociacionismo en España" En Revista Española de Ciencia Política. Núm. 13, Octubre 2005, p. 51-87.

NIVOLA, P. S., \& BRADY, D. W. (Eds.). (2008). Red and Blue Nation?: Consequences and Correction of America's Polarized Politics (Vol. 2). Brookings Institution Press.

NORRIS, P. (2000) A Virtuous Circle: Political Communication in Post-Industrial Societies. NY: Cambridge Press, Fall.

REY, P. (2013) "El color de la corrupción, en periódicos españoles y catalanes". En Capçalera del Col-legi de Periodistes de Catalunya. Junio 2013, urL: http:// numeroteca.org/2013/07/09/el-color-de-la-corrupcion/ capturado el 12 de enero de 2014.

SAMPEDRO BLANCO, V. (Ed) (2005). 13M, Multitudes on line. Madrid: Ediciones Los libros de la Catarata.

SAMPEDRO, V. y SEOANE PÉREZ, F. (2008). "The 2008 Spanish general elections: "Antagonistic bipolarization" geared by presidential debates, partisanship, and media interests". En The International Journal of Press/Politics, vol. 13 (3). P. 336-344.

SAMPEDRO BLANCO, V. (ed.) (2008) Medios y elecciones 2004. La campaña electoral y las "otras campañas" Madrid: Centro de Estudios Ramón Areces.

TERUEL RODRÍGUEZ, L. (2006). Los medios en la construcción de la realidad política: el caso de El Mundo (1993-1996). Málaga: Servicio de Publicaciones de la Universidad de Málaga.

TERUEL RODRÍGUEZ, L. (2013). "El posicionamiento editorial de la prensa española sobre la polarización política" En CAPDEVILA, A., CANEL CRESPO, MJ., GURRIONERO, M. y SANDERS, K. (eds) (2013) Estudios De Comunicación Política. Libro Del Año 2012 Ed. Tecnos. 978-84-309-5530-5. P. 169-191.

TERUEL RODRÍGUEZ, L. (2014) "La prensa española ante el nacimiento de nuevas fuerzas políticas: ¿el fin del bipartidismo?". En Contenidos Especializados en la enseñanza superior. ISBN 978-84-15705-27-7.

THOMPSON, J. (2000). El escándalo político. Poder y visibilidad en la era de los medios de comunicación. Barcelona: Ediciones Paidós Ibérica.

7.1 Recursos Web

HUMANES, M.L., MONTERO SÁNCHEZ, M.D., MOLINA DE DIOS, R. Y LÓPEZ-BERINI, A (2013): "Pluralismo y paralelismo político en la información televisiva en España", en Revista Latina de Comunicación Social. URL http:// www.revistalatinacs.org/068/paper/990_Fuenlabrada/24_Humanes.html

LLANERAS, K. (2015) "Los problemas de los españoles antes y después de la crisis". En Politikon. URL http://politikon.es/2015/01/21/los-problemas-de-losespanoles-antes-y-despues-de-la-crisis-2/ Capturado el 2 de febrero de 2015. 
MARTÍNEZ NICOLÁS, M. (2001) "De la brecha digital a la brecha cívica. Acceso a las tecnologías de la comunicación y participación ciudadana en la vida pública". En Revista Telos. Núm 86, enero-marzo 2011. http://telos.fundaciontelefonica.com/DYC/TELOS/NMEROSANTERIORES/Nmeros8097/ DetalleAnteriores 86TELOS PERSPECT 1/seccion=1268\&idioma=es ES\&i$\mathrm{d}=2011012708250001$ \&activo $=6$.do

RODRÍGUEZ-CASTROMILA, A.; RODRÍGUEZ DÍAZ, R. (2011) "Terrorismo con y sin tregua. Políticos, ciudadanos y medios de comunicación". En Revista Telos. No 87 Abril-junio 2011. P1-10. http://telos.fundaciontelefonica.com/DYC/ TELOS/NMEROSANTERIORES/Nmeros8096/DetalleAnteriores 87TELOS PERSPECT_3 $/$ seccion $=1268 \&$ idioma $=$ es_ES\&id $=2011051309530001 \&$ activo $=6$. do

RODRÍGUEZ-POLO, X. R. (2013). "Bloqueo mediático, redes sociales y malestar ciudadano. Para entender el movimiento español del 15-M". En Palabra Clave, $\mathrm{n}^{\mathrm{o}} 16$ (1), p. 45-68. URL http://www.scielo.org.co/scielo.php?pid=S0122$82852013000100003 \&$ script $=$ sci_arttext\&tlng=en

\section{Notas}

1 Se han estimado los espacios informativos al completo -puesto que el deporte tiene un programa propio- y una parte proporcional de los magazines tanto en La Sexta (Más Vale Tarde), como en Tele5 (Ana Rosa) y Antena 3 (Espejo Público) pues todos los contenidos de estos últimos no son políticos sino que también abarca sociedad, sucesos, corazón... Se ha estimado que la emisión diaria de las cadenas es de veinte horas.

2 En la escala entre 1-izquierda y 10-derecha, laSexta obtuvo un 5,9 y Antena 3 un 3,8

3 Más aún, dentro de su target comercial (personas de 13 y 54 años, sin incluir clase baja, que viven en poblaciones de más de 10.000 habitantes) se eleva hasta el 17,2\%. Un dato muy por encima de sus rivales en la franja de competencia: Antena 3 (11,4\%), Cuatro (10,8\%), La 1 (9,5\%), Telecinco $(8,6 \%)$.

4 Véase “Antonio Caño rechaza que 'El País' sea próximo al PSOE y niega pactos con Zarzuela” URL http://vozpopuli.com/economia-y-finanzas/42826-antonio-cano-rechaza-que-el-pais-seaproximo-al-psoe-y-niega-pactos-con-zarzuela Capturado el 8 de enero de 2015.

5 Así, por ejemplo, las tres noticias relacionadas con esta formación más leídas en $A b c$, según su hemeroteca digital, son: "Así sería un país gobernado por Podemos" (24-05-2014) -que alude al fin de la política de seguridad ciudadana-, "Podemos ya es un negocio" (03-02-2015) y "Una jauría tuitera defiende a Podemos de sus críticos en las redes" (04-02-2015). Por su parte, El País ha publicado una serie de reportajes de John Carlin en enero de 2015-“¿Qué quiere Podemos?- el primero de los cuales sostiene que se trata de un movimiento populista y que: "La sabiduría de las masas es un concepto cuestionable, muchas veces basado en la ignorancia o en la histeria colectiva". 